\title{
Vortex formation in a cavity with oscillating walls
}

\author{
G. Ovando ${ }^{\mathrm{a}, *}$ H. Juárez ${ }^{\mathrm{b}}$, G. Huelsz ${ }^{\mathrm{a}}$ and E. Ramos ${ }^{\mathrm{a}, \mathrm{c}}$ \\ ${ }^{a}$ Centro de Investigación en Energía, UNAM, Ap. P. 34, Temixco, Mor. 62580, Mexico \\ ${ }^{\mathrm{b}}$ Departamento de Matemáticas UAM-I, Ap. P. 55-534, 09340 Mexico D.F. Mexico \\ ${ }^{\mathrm{c}}$ Max Planck Institute for the Physics of Complex Systems, Noethnitzer Strasse 38, 01187 Dresden, Germany
}

June 9, 2008

\begin{abstract}
The vortex formation in a two-dimensional Cartesian cavity, which their vertical walls move simultaneously with an oscillatory velocity and the horizontal walls are fixed pistons, is studied numerically. The governing equations were solved with a finite element method combined with an operator splitting scheme. We analyzed the behavior of vortical structures occurring inside a cavity with an aspect ratio of height to width of 1.5 for three different displacement amplitudes of the vertical oscillatory walls (amplitude/width $=Y=0.2,0.4$ and 0.8 ) and Reynolds numbers based on the cavity width of 50,500 and 1000. Two vortex formation mechanisms are identified: a) the shear, oscillatory motion of the moving boundaries coupled with the fixed walls that provide a translational symmetry-breaking effect and b) the sharp changes in the flow motion when the flow meets the corners of the cavity. The vortices cores were identified using the Jeong-Hussain criterium and it is found that they occupy smaller areas as the Reynolds number increases. All flows studied are cyclic symmetric and for low $Y$ and $R e$ values they are also symmetric with respect to the vertical axis dividing the cavity in two sides. In asymmetric flows, the unbalance between the vortices on each side of the mid-vertical line generates a vortex that occupies the central part of the cavity. The breakdown of the axial symmetry was studied for a fixed value of the oscillation amplitude, $Y=0.8$, taking $R e$ as the bifurcation parameter. The results indicates that the symmetry is broken through a supercritical pitchfork bifurcation.
\end{abstract}

\section{Introduction}

The oscillatory flow generated by the relative periodic motion of the walls containing a fluid has been studied in detail due to the numerous technologically relevant situations where these conditions occur. Perhaps the most important application is the problem of a piston moving in a cylindrical container as it happens

${ }^{*}$ Corresponding author. Tel.:+52+55+562-29711; Fax:+52+55+562-29741; E-mail address: geoc@cie.unam.mx 
in internal combustion engines [1], but other examples like a device for measuring the surface dilatational viscosity of a gas/liquid interface [2] have motivated other important aspects of the analysis as well. A study closely related to the present investigation was reported by O'Brien [3]. The analysis consisted in numerically solving a model for the flow generated by the in-phase sinusoidal motion of two opposite walls of a two dimensional container with aspect ratio of 0.5 . The flow is assumed to be symmetric and the emphasis of the study was on the unsteady separation.

Studies have been done both for a moving cylinder wall with fixed piston, or for a moving piston. According to Tabaczynski et al. [4], the flow near the corner is equivalent in both cases provided $\sqrt{\nu t} / U_{w} T \ll 1$, where $\nu$ is the kinematic viscosity, $t$ is the time, $U_{w}$ is the instantaneous piston speed, and $T$ is the period. The pioneering experimental observations of Tabaczynski et al. [4] have shown that the fluid motion is a sink-type flow when the cylinder wall retreats from the piston and a spiral ring vortex motion near the piston face and cylinder wall interface when the cylinder wall moves toward the piston, both for constant and oscillatory velocity of the walls. The flow in the vicinity of the corner as the wall moves towards a fixed piston with a steady motion was studied theoretically by Taylor [5] and Batchelor [6], assuming that the inertial effects can be neglected. In this region, the velocity gradients are large and a discontinuity exists in the velocity field at the junction of the moving piston relative to the wall. Also, close to the corner, viscous forces are much larger than inertial ones. Using an order of magnitude argument Batchelor [6] indicates that the distance $r$ from the corner to where a steady viscous solution is applicable is of the order of $r \ll \nu / U_{w}$, but experiments of Allen and Chong [7] have shown that the validity of the viscous solution is significantly larger than $\nu / U_{w}$.

Tabaczynski et al. [4] and Allen and Chong [7] studied the dimension of the vortex generated when the lateral wall moves towards the piston. The first authors considered constant and sinusoidal wall speed while Allen and Chong [7] observed the phenomenon for constant or power law wall speed. Analysis and numerical studies on incompressible flows are done considering isothermal conditions.

In a series of reports, Lopez and collaborators [8], [9], [10] analyzed the flow in a rectangular cavity driven by the sinusoidal motion of one of the walls in its own plane. The main objective was the identification of the stability limits of the time-periodic, two-dimensional base state. Their results include the determination of regions in the two-dimensional (Re,St) parametric space. The system may adopt one of three possible flow regimes: an essentially two-dimensional time-periodic flow, a time-periodic three-dimensional flow, and a three-dimensional, space and time irregular flow. In the limit where the spanwise direction is infinite, the range of parameters explored was $500<R e<1400$ and $0<S t<200$ and Their results indicate that for flows with $R e<520$, the flow is two dimensional, and that the locus of the bifurcation points from two-dimensional to three-dimensional flows displays a local minimum at $R e=520, S t=20$. For $S t>20$, the locus of the bifurcation points roughly follows roughly Re is proportional to St.

In this work, we analyzed the vortices formation in all the cavity with vertical oscillating walls, for $R e=50,500$ and 1000 with $Y=0.2,0.4$ and 0.8. An important result is the identification of a two 


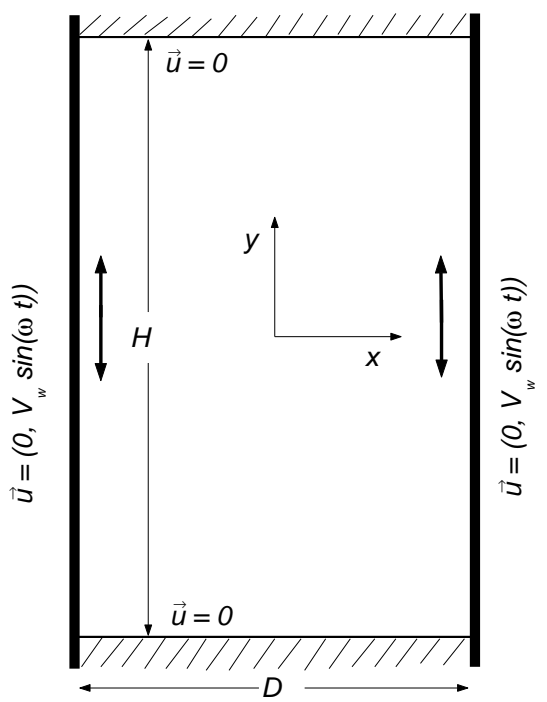

Figure 1: Geometry of the container with sliding vertical walls and boundary conditions.

dimensional instability in the flow at relatively large amplitude of the wall motion and Reynolds numbers.

\section{Problem formulation}

\subsection{Physical and geometry description}

We present numerical simulations in two dimensional cavity with a ratio of 1.5 between vertical and horizontal dimensions as shown in figure (1). The vertical walls move simultaneously with an oscillatory velocity while the horizontal walls are the fixed pistons, similar to the experimental arrangement used by Tabaczynski et al. [4]. For water as the working fluid, the vertical and horizontal dimensions of the cavity are $H=7.5 \times 10^{-2} \mathrm{~m}$ and $D=5.0 \times 10^{-2} \mathrm{~m}$, respectively.

\subsection{Governing equations}

Considering a two dimensional region $\Omega$, where the flow of the cavity is analyzed, the conservation equations that describe the oscillatory flow of an incompressible fluid in this region are the Navier-Stokes equation and the continuity equation:

$$
\begin{aligned}
\frac{\partial \vec{u}}{\partial t}-\nu \Delta \vec{u}+\vec{u} \cdot \nabla \vec{u}+\nabla p & =0 \text { in } \Omega \times\left[0, t_{f}\right], \\
\nabla \cdot \vec{u} & =0 \text { in } \Omega \times\left[0, t_{f}\right],
\end{aligned}
$$

where $\vec{u}=\left(u_{1}, u_{2}\right)$ is the velocity vector, being $u_{1}$ and $u_{2}$ the transversal and axial velocity components respectively; $\nu$ is kinematic viscosity, $p$ is the pressure, $t$ is the time and $t_{f}$ is the final time. 
The boundary conditions of the oscillatory cavity are:

$$
\begin{aligned}
& u_{1}(x=-D / 2, y, t)=0, \quad u_{2}(x=-D / 2, y, t)=V_{\mathrm{W}} \sin \omega t, \\
& u_{1}(x=D / 2, y, t)=0, \quad u_{2}(x=D / 2, y, t)=V_{\mathrm{W}} \sin \omega t, \\
& u_{1}(x, y=-H / 2, t)=0, \quad u_{2}(x, y=-H / 2, t)=0 \\
& u_{1}(x, y=H / 2, t)=0, \quad u_{2}(x, y=H / 2, t)=0
\end{aligned}
$$

$V_{\mathrm{W}}$ is the amplitude of the oscillatory velocity of the vertical walls given by $V_{\mathrm{W}}=Y_{\mathrm{W}} \omega$, where $\omega$ is the frequency and $Y_{\mathrm{W}}$ is the displacement amplitude.

\section{$3 \quad$ Numerical method}

\subsection{Weak formulation of the problem}

Since we want to approximate the variables $\vec{u}$ and $p$ by finite element method, we need to obtain the so called weak formulation of equations (1-2). For this purpose we introduce the following notation: Let $\Gamma$ be the boundary of the domain $\Omega$, and $\Gamma=\Gamma_{\mathrm{W}} \cup \Gamma_{\mathrm{p}}$, where $\Gamma_{\mathrm{w}}$ represents the two vertical sliding walls and $\Gamma_{\mathrm{p}}$ represents the two horizontal fixed walls. Let $\vec{g}_{0}(\vec{X}, t)$ be a vector defined by

$$
\vec{g}_{0}(\vec{X}, t)=\left\{\begin{array}{llll}
\overrightarrow{0} & \text { if } & \vec{X} \epsilon \Gamma_{\mathrm{p}}, & t \epsilon\left(0, t_{f}\right), \\
\left(0, V_{\mathrm{W}} \sin \omega t\right) & \text { if } & \vec{X} \epsilon \Gamma_{\mathrm{W}}, & t \epsilon\left(0, t_{f}\right) .
\end{array}\right.
$$

We introduce the following sets of functions:

$$
\begin{gathered}
L_{2}(\Omega)=\left\{q: \int_{\Omega} q^{2} d \vec{X}<\infty\right\}, \\
\vec{V}=V \times V,
\end{gathered}
$$

where $V=H^{1}(\Omega)=\left\{v \in L_{2}(\Omega): \frac{\partial v}{\partial x_{i}} \in L_{2}(\Omega), \quad i=1,2\right\}$.

We also introduce the following spaces of functions:

$$
\begin{gathered}
L_{0}=\left\{q \in L_{2}(\Omega): \int_{\Omega} q(\vec{X}) d \vec{X}=0\right\}, \\
\vec{V}_{0}=\{\vec{v} \epsilon \vec{V}: \vec{v}=\overrightarrow{0} \text { on } \Gamma\} .
\end{gathered}
$$

Applying the virtual power principle to the momentum equation and to the continuity equation, we obtain:

$$
\begin{gathered}
\int_{\Omega}\left[\frac{\partial \vec{u}}{\partial t} \cdot \vec{v}+(\vec{u} \cdot \nabla) \vec{u} \cdot \vec{v}\right] d \vec{X}+\nu \int_{\Omega} \nabla \vec{u}: \nabla \vec{v} d \vec{X}-\int_{\Omega} p \nabla \cdot \vec{v} d \vec{X}=0, \quad \forall \quad \vec{v} \epsilon \vec{V}_{0}, \\
\int_{\Omega} q \nabla \cdot \vec{u} d \vec{X}=0, \quad \forall \quad q \epsilon L_{2}(\Omega), \\
\vec{u}(\vec{X}, t)=\vec{g}_{0}(\vec{X}, t) \quad \text { on } \quad \Gamma \times\left(0, t_{f}\right) .
\end{gathered}
$$


These equations are completed by the following initial conditions

$$
\vec{u}(\vec{X}, 0)=\vec{u}_{0}
$$

In the above weak formulation, it is reasonable to assume that $\vec{u}(t) \epsilon \vec{V}$ and $p(t) \epsilon L_{0}$ (see [11], [12], [13] and [14]), where we use the notation $\vec{u}(t) \equiv \vec{u}(\vec{X}, t) \quad \forall \vec{X} \epsilon \Omega$ and $t$ fixed. Similarly for $t>0$ fixed, $p(t) \equiv p(\vec{X}, t) \forall \vec{X} \epsilon \Omega$.

\subsection{Finite element approximation}

Let $h$ be a space discretization step and $\tau_{h}$ a finite element triangulation of the polygonal domain $\bar{\Omega}$. Let $P_{1}$ be the space of polynomials in two variables of degree less or equal to one, and let $\mathcal{C}^{0}(\bar{\Omega})$ be the space of continuous functions defined in $\bar{\Omega}$. We construct another finite element triangulation $\tau_{h / 2}$ of $\bar{\Omega}$ which is twice thinner than $\tau_{h}$, by subdividing each triangle of $\tau_{h}$ into four similar subtriangules by the mid point sides as shown in figure (2).

Then we approximate the spaces introduced in the previous section by the following finite dimensional spaces, respectively:

$$
\begin{gathered}
L_{h}=\left\{q_{h}\left|q_{h} \in \mathcal{C}^{0}(\bar{\Omega}), \quad q_{h}\right|_{T} \in P_{1}, \quad \forall \quad \tau \epsilon \tau_{h}\right\}, \\
L_{0 h}=\left\{q_{h} \epsilon L_{h} \mid \int_{\Omega} q_{h} d \vec{X}=0\right\}, \\
V_{h}=\left\{\vec{v}_{h}\left|\vec{v}_{h} \epsilon\left(\mathcal{C}^{0}(\bar{\Omega})\right)^{2}, \quad \vec{v}_{h}\right|_{T} \epsilon P_{1} \times P_{1}, \quad \forall \quad \tau \epsilon \tau_{h / 2}\right\}, \\
V_{0 h}=\left\{\vec{v}_{h} \epsilon V_{h} \mid \vec{v}_{h}=\overrightarrow{0} \quad \text { on } \quad \partial \Omega_{1}\right\} .
\end{gathered}
$$

This approximation was derived from the so called Bercovier-Pironneau ( or P1 iso P2/P1) finite element approximation which was introduced in [15] for the velocity-pressure formulation of the Stokes problem. In the above approximation the degrees of freedom for the velocity of the fluid are located on the vertices of each triangle $\tau_{h / 2}$, while the degrees of freedom for the pressure are located on the vertices of each triangle in $\tau_{h}$, as shown in figure (2). Using the above finite dimensional spaces leads to the following approximation of equations (3-6).

For $t>0$, find $\vec{u}_{h}(t) \epsilon V_{h}$ with $\vec{u}_{h}(t)=\vec{g}_{0 h}(t)$ on $\Gamma, p_{h} \epsilon L_{0 h}$ such that

$$
\begin{gathered}
\int_{\Omega}\left[\frac{\partial \vec{u}_{h}}{\partial t} \cdot \vec{v}+\left(\vec{u}_{h} \cdot \nabla\right) \vec{u}_{h} \cdot \vec{v}\right] d \vec{X}+\nu \int_{\Omega} \nabla \vec{u}_{h}: \nabla \vec{v} d \vec{X}-\int_{\Omega} p_{h} \nabla \cdot \vec{v} d \vec{X}=0, \quad \forall \quad \vec{v} \epsilon V_{0 h}, \\
\int_{\Omega} q \nabla \cdot \vec{u}_{h} d \vec{X}=0, \quad \forall \quad q \epsilon L_{h}, \\
\vec{u}_{h}(\vec{X}, 0)=\vec{u}_{0 h}(\vec{X}) .
\end{gathered}
$$

In the previous discretization $\vec{g}_{0 h}$ is the finite element approximation of the boundary function $\vec{g}_{0}$, with must satisfy $\int_{\Gamma} \vec{g}_{0 h} \cdot \vec{n} d \Gamma=0$. Similarly, $\vec{u}_{0 h}$ is finite element approximation of the initial condition function $\vec{u}_{0}$. 

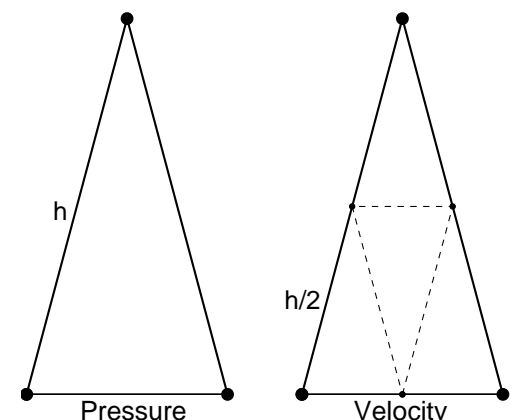

Figure 2: Degrees of freedom for pressure and velocity in the finite element approximation.

\subsection{Time discretization by operator splitting}

Following Chorin [16], most modern Navier-Stokes solvers are based on operator splitting schemes (see [17] and [18]) in order to force the incompressibility condition via Stokes solver or a $L^{2}$-projection method. This approach still applies to the initial value problem 7-9 which contain three numerical difficulties to each of which can be associated a specific operator, namely

a) The incompressibility condition (8) and the related unknown pressure (7)

b) The advection term in (7)

c) The diffusion term in (7)

From an abstract point of view, problem 7-9 is a particular case of the following class of initial value problems:

$$
\frac{d \Theta}{d t}+\sum_{i=1}^{3} A_{i}(\Theta, t)=f, \quad \Theta(0)=\Theta_{0},
$$

where the operators $A_{i}$ can be multivalued. There are many operator-splitting methods that can be employed to solve this type of problems. Here we consider the fractional step scheme of Marchunk-Yaneuko [19] type: Let $\Delta t>0$ be a time discretization step. Given $\Theta^{0}=\Theta_{0}$, compute $\Theta^{n+1}$ from $\Theta^{n}$ by solving:

$$
\begin{gathered}
\frac{\Theta^{n+\frac{i}{3}}-\Theta^{n+\frac{i-1}{3}}}{\Delta t}+A_{i}\left(\Theta^{n+\frac{i}{3}}, t^{n+1}\right)=f_{i}^{n+1}, \\
\text { for } i=1,2,3 \quad \text { with } t^{n}=n \Delta t, \quad \text { and } \quad \sum_{i=1}^{3} f_{i}^{n+1}=f^{n+1} .
\end{gathered}
$$

This very simple scheme is only first order accurate (see [19]), but its low order is compensated by good stability and robustness properties. Actually, this scheme can be made second order accurate by symmetrization (see [20] and [21] for the application of symmetrized splitting schemes to the solution of the Navier-Stokes equations). 
Applying scheme (11) to the equations (7-9), we obtain the following three stage scheme (after dropping some of the subscripts h), given $\vec{u}^{0}=\vec{u}_{0 h}$ and assuming we know $\vec{u}^{n}$ for $n \geq 0$ :

Step 1. Find $\vec{u}^{n+\frac{1}{3}} \epsilon V_{h}$ with $\vec{u}^{n+\frac{1}{3}}=\vec{g}_{0 h}^{n+1}$ on $\Gamma$, and $p^{n+\frac{1}{3}} \epsilon L_{0 h}$ such that

$$
\begin{gathered}
\int_{\Omega} \frac{\vec{u}^{n+\frac{1}{3}}-\vec{u}^{n}}{\Delta t} \cdot \vec{v} d \vec{X}+\int_{\Omega} p^{n+\frac{1}{3}} \nabla \cdot \vec{v} d \vec{X}=0, \quad \forall \quad v \in V_{0 h}, \\
\int q \nabla \cdot \vec{u}^{n+\frac{1}{3}} d \vec{X}=0, \quad \forall \quad q \in L_{h} .
\end{gathered}
$$

Step 2. Find $\vec{u}^{n+\frac{2}{3}}=\vec{u}\left(t^{n+1}\right)$, where $\vec{u}(t)$ is the solution on $\left(t^{n}, t^{n+1}\right)$ of the following advection problem

$$
\int_{\Omega} \frac{\partial \vec{u}(t)}{\partial t} \cdot \vec{v} d \vec{X}+\int_{\Omega}\left(\vec{u}^{n+\frac{1}{3}} \cdot \nabla\right) \vec{u}(t) \cdot \vec{v} d \vec{X}=0, \quad \forall \quad v \epsilon V_{0 h}^{-},
$$

$\vec{u}\left(t^{n}\right)=\vec{u}^{n+\frac{1}{3}}$, and $\vec{u}(t)=\vec{g}_{0 h}\left(t^{n+1}\right)$ on $\Gamma^{-} \times\left(t^{n}, t^{n+1}\right)$, where $\Gamma_{1}^{-}=\left\{\vec{x} \epsilon \Gamma \mid \vec{g}_{0 h} \cdot \vec{n}(\vec{X})<0\right\}$ and $V_{0 h}^{-}=$ $\left\{\vec{v} \epsilon V_{h} \mid \vec{v}=0\right.$ on $\left.\Gamma^{-}\right\}$.

Step 3. Find $\vec{u}^{n+1} \epsilon V_{h}$ with $\vec{u}^{n+1}=\vec{g}_{0 h}^{n+1}$ on $\Gamma$ such that

$$
\int_{\Omega} \frac{\vec{u}^{n+1}-\vec{u}^{n+\frac{2}{3}}}{\Delta t} \cdot \vec{v} d \vec{X}+\frac{1}{R e} \int_{\Omega} \nabla \vec{u}^{n+1}: \nabla \vec{v} d \vec{X}=0, \quad \forall \quad \vec{v} \epsilon V_{0 h} .
$$

Problem (12) is a finite dimensional problem known as a saddle-point system which was solved by a Uzawa/conjugate-gradient algorithm discussed with many details in, e.g. refs. [14] and [22]. Problem (14) is a discrete elliptic system whose iterative or direct solution is a quite classical problem. In this work this elliptic system was solved by a conjugate gradient method adapted for sparse systems [23]. On the other hand, solving the pure advection equation (13) is a more delicate issue. This equation can be solved by a method of characteristics as in [17] and [24]. An easier alternative is provided by the wave-like equation method discussed in [14], [21] and [25]. We applied this last method to solve equation (13).

\subsection{Mesh convergence analysis}

The simulations were made with three different meshes for all cases, the first one was an equidistant mesh with a constant spacial step $\Delta x$ in the transversal direction, and a constant spacial step $\Delta y$ in the axial direction. The second one was a graded mesh, such that the refinement in the corner of the domain was $1 / 3 \Delta x$ and $1 / 3 \Delta y$. The third one was a graded mesh, such that the refinement in the corner of the domain was $1 / 5 \Delta x$ and $1 / 5 \Delta y$. For $R e=50$ we used a mesh with $41 \times 51$ subdivisions to approximate pressure, for $R e=500$ we used a mesh with $81 \times 101$ subdivisions, and for $R e=1000$ we used a mesh with $101 \times 121$ subdivisions. To approximate velocity we used twice as finer meshes for each case. Analyzing the convergence of the three meshes for stationary state we could observed important differences between the results obtained with the $1 / 3$ graded mesh and the equidistant mesh, however the values obtained with the $1 / 5$ and $1 / 3$ graded mesh are practically the same, this behavior was observed for all cases. In figure (3), it can be observed an example of the mesh convergence. The graded meshes were generated such that the 


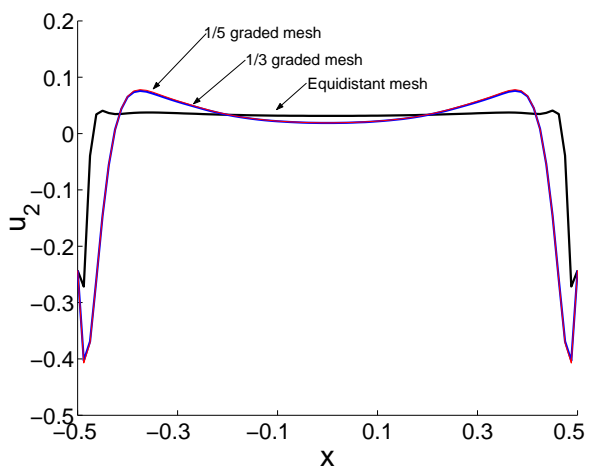

Figure 3: Profile of axial velocity $u_{2}$ as a function of the transversal coordinate $x$. For the equidistant, $1 / 3$ graded and $1 / 5$ graded meshes, for $R e=500$ and $Y=0.4$ in $y=0.15$.

spacial step increased from the corners to the center in both direction.

In the transversal direction we used the law

$$
x_{k}=x_{k-1}+r_{x}^{k-2} \epsilon_{x} \quad \forall 2 \leq k \leq n c,
$$

and

$$
x_{n c+j}=D-x_{n c-j} \quad \forall 1 \leq j \leq n c-1,
$$

with $r_{x}$ being the increasing common ratio, and

$$
\epsilon_{x}=\left(r_{x}-1\right) /\left(r_{x}^{n l}-1\right)(D / 2),
$$

where $n l=$ elements number in the $\mathrm{x}$-direction $/ 2, n c=(n-1) / 2+1$ with $n=$ nodes number in the $\mathrm{x}$-direction. In the axial direction a similar law was used.

For all cases the worst relative error for axial and transversal velocities between the $1 / 5$ and $1 / 3$ graded meshes were, in more than $95 \%$ of the domain, less than $1.5 \%$ and $3 \%$, respectively. In figure (4), is shown the worst relative error for axial velocity.

\section{$4 \quad$ Results}

\subsection{Overview}

To initiate the study of the vortex formation in the cavity with oscillating walls, simulations for nine cases were done for $R e=V_{\mathrm{W}} D / \nu=50,500,1000$, and $Y=0.2,0.4,0.8$. We characterize the flows by describing the velocity fields and vortices cores. The vortices cores were obtained by applying the Jeong-Hussain criterium [26], which defines a vortex in an incompressible flow in terms of the eigenvalues of the tensor $S^{2}+A^{2}$, where $S$ and $A$ are respectively the symmetric and antisymmetric parts of the velocity gradient tensor. In planar flow, a vortex core is defined as the area where the eigenvalue of $S^{2}+A^{2}$ is negative. 


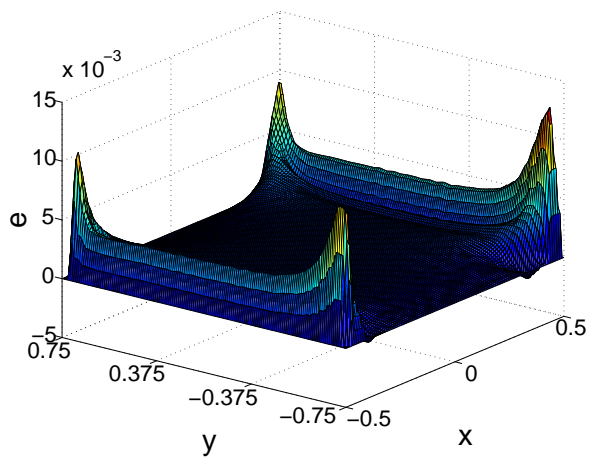

Figure 4: Relative error for axial velocity as a function of the position $(x, y)$ between $1 / 5$ and $1 / 3$ graded meshes, for $R e=500$ and $Y=0.4$.

As expected from the governing equations and boundary conditions, the velocity fields and consequently the vortices cores for low $R e$ and $Y$ display axial and cyclic symmetries, defined as follows:

a) axial symmetry

$$
u_{1}(x, y, \phi)=-u_{1}(-x, y, \phi), \quad u_{2}(x, y, \phi)=u_{2}(-x, y, \phi)
$$

and

b) cyclic symmetry

$$
u_{1}(x, y, \phi)=u_{1}(x,-y, \phi+\pi), \quad u_{2}(x, y, \phi)=-u_{2}(x,-y, \phi+\pi) .
$$

In fact, among the first nine simulated cases, only axial symmetry is lost for $R e=1000$ and $Y=0.8$.

In order to have a convenient reference for interpreting the dynamics of the flow, figure (5) emphasizes that the velocity and the displacement of the vertical walls are one quarter of a cycle out of phase. We see for instance that for $\phi=0$, the velocity of the vertical walls of the cavity is zero while the displacement is maximum negative and for $\phi=\pi / 2$, the velocity of the vertical walls of the cavity is maximum positive while the displacement is zero. Due to the symmetries of the flow, we can give a fairly complete account of the important features of the motion by describing the flows at these two representative phases.

The flow produced by two opposite, oscillating walls in a finite aspect ratio channel generates vortex motion due to various mechanisms. Two of them determine the most salient features of the flow and we describe them here somewhat in detail. The first is vorticity injection into the fluid due to the shear motion of the moving boundaries and a translational symmetry-breaking mechanism due to the presence of the pistons. The oscillatory boundary acts as vorticity source and the vortices generated are attached to moving walls. This process is labeled $M 1$. The second vortex generation mechanism (labeled $M 2$ ) is the sharp change in the flow direction as the fluid set in motion by the moving walls meets the fixed wall. The main physical 


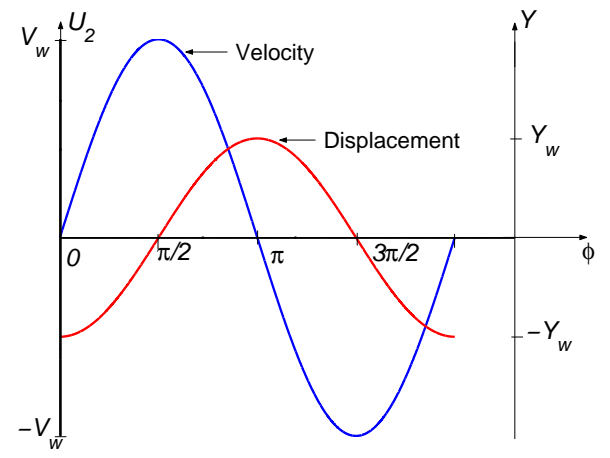

Figure 5: Velocity and displacement of vertical walls as a function of phase.

process in this case consists on roll up of cast off vortex sheets and has been discuscussed by Tabaczynski et al. [4] and Allen and Chong [7] for large Reynolds numbers. Vortices generated by $M 1$ are noticeable along the whole cycle, they change their vorticity sign along the cycle and are located in the regions near the moving walls. In contrast, $M 2$ generates vortices near horizontal walls when the fluid impinges on them; therefore, it is more noticeable on specific phases, but they do not change their vorticity sign along the cycle. For instance, vortices are generated via $M 2$ near the lower horizontal wall for $\phi=0$ and near the higher horizontal wall for $\phi=\pi$.

\subsection{Case $R e=50$}

Figure (6) shows the velocity and vorticity fields for $R e=50$ and $Y=0.2$, for two phases of the oscillation. In this vorticity field and the followings, black lines limit the vortex cores according to JeongHussain criterium. At $\phi=0$ when the velocity of the walls is zero and they are at their lowermost position, the flow is organized in two vortices elongated in the vertical direction formed by downward moving boundary layer near the vertical walls that turn at the bottom and form a central upward flow. At this phase, the left red vortex is anticlockwise (+) while the right blue vortex is clockwise (-). The geometry of the vortical structures as revealed by the Jeong-Hussain criterium, indicate that vortices are thicker at the bottom due to the merging of the vortices generated by the concurrence of the two mechanisms described in the previous section (M1 and M2). This is the region of larger vorticity. This flow originates half saddle critical points at the centers of the horizontal walls. Two elliptical points are identified at $x= \pm 0.32, y=0$ indicating the center of the vortices.

The flow at $\phi=\pi / 2$ displays thin ascending regions near the moving walls which at this phase of the cycle have maximum positive velocity. The returning, descending flows are confined to regions close to the vertical walls and in the core of the container, the fluid is practically stagnant, at this phase the left vortex is clockwise (-) while the right vortex is anticlockwise $(+)$. The vortices generated by the flow at this phase, display an almost constant thickness and are located close to the moving walls, as shown in figure (6). The 
vortices at this phase of the cycle, are gererated by mechanism $M 1$. As expected, the vortices are located around elliptic points and away from saddle points. The vertical extension of the vortices remain almost constant through the cycle, but the thickness pulsates with maxima (minima) at $\phi=0, \pi(\phi=\pi / 2,3 \pi / 2)$. The effect of increasing the amplitude of the wall displacement $(Y)$ is illustrated in figure (7), where velocity fields and vortices are shown for $R e=50$ and $Y=0.4$. The most notable difference between the flows in figures (6) and (7) is that the thickness of the vortical structure increases as the amplitude increases. This is expected since the moving walls constitute a vorticity source. When the amplitude of the oscillation is further increased to $Y=0.8$, the thickening of the vortices continues and in this case, the vortices cover at least $50 \%$ of the total area. This is illustrated in figure (8) where the velocity field and vortices cores are shown.

In order to assess the influence of the top and bottom fixed walls on the dynamics of the flow, we analyze the profile of the axial velocity $u_{2}$ as a function of the transversal coordinate for several phases in one cycle. For reference, we recall that the velocity profile as a function of the transversal coordinate in an infinitely long channel with oscillating walls (Second Stokes Problem, see reference [27]) is given by

$$
u(x, t)=B V_{\mathrm{W}} \cos \omega t+C V_{\mathrm{W}} \sin \omega t
$$

where

$$
B=\left[\frac{\cos l \beta \cos x \beta \cosh l \beta \cosh x \beta+\sin l \beta \sin x \beta \sinh l \beta \sinh x \beta}{(\cos l \beta \cosh l \beta)^{2}+(\sin l \beta \sinh l \beta)^{2}}\right],
$$

and

$$
C=\left[\frac{\cos l \beta \sin x \beta \cosh l \beta \sinh x \beta-\sin l \beta \cos x \beta \sinh l \beta \cosh x \beta}{(\cos l \beta \cosh l \beta)^{2}+(\sin l \beta \sinh l \beta)^{2}}\right],
$$

where $l=D / 2$ and the parameter $\beta$ is the inverse of the Stokes penetration depth and is defined by

$$
\beta=\sqrt{\frac{\omega}{2 \nu}},
$$

where $\omega$ is the angular velocity, and $\nu$ is the kinematic viscosity. This velocity profile is shown in figure (9) taking $R e=50$ and $Y=0.2$. It is seen that for these conditions, the Stokes penetration depth is smaller than the semi distance between the channel walls $\left((D / 2) / \delta_{\nu}=5.5\right)$ and for positive (or negative) $x$, the envelopes are monotonous functions of this coordinate. The flow in the region near the center of the channel $(x \sim 0)$ is practically stagnant at all times. Also, it should be noted that for an infinite channel, the $x$-averaged instantaneous velocity is not zero, while for the finite aspect ratio channel, the $x$-averaged instantaneous velocity must be zero at all times due to presence of the horizontal walls.

The equivalent velocity profiles obtained at $y=0$, for the channel with finite aspect ratio $(H / D=1.5)$ are shown in figure (10). The envelopes are not monotonous functions of $x$. In contrast to the infinite aspect ratio channel, the flow at the center of the channel is stagnant only for specific phases in the cycle. In the region close to the lateral walls $\left(\delta_{\nu}\right)$, the velocity profiles for infinite and finite aspect ratios coincide, indicating that 
at $y=0$, the presence of the horizontal walls is felt only in the core of the channel. When the amplitude of the oscillatory motion of the walls is increased to $Y=0.8$, the features just described are amplified as can be observed in figure (11). Another useful parameter to describe the effect of the presence of the horizontal walls on the dynamics of the oscillatory flow is the phase shift of the fluid oscillation as a function of the distance to the vertical wall $\psi$. As is well known, in the Second Stokes Problem, the fluid oscillates with a phase shift linearly proportional to the distance to the wall. The proportionality constant is $\beta$. For the infinite channel, the phase shift follows from equation (15) and is:

$$
\psi=\operatorname{arctag}\left[\frac{\cos l \beta \sin x \beta \cosh l \beta \sinh x \beta-\sin l \beta \cos x \beta \sinh l \beta \cosh x \beta}{\cos l \beta \cos x \beta \cosh l \beta \cosh x \beta+\sin l \beta \sin x \beta \sinh l \beta \sinh x \beta}\right] .
$$

In figure $(12),(\psi)$ is shown as a function of the transversal coordinate for $y=0$ and $R e=50$. Two cases are displayed, $Y=0.2$ and 0.8; the lines are for an infinite long channel (equation (16)), while symbols represent finite aspect channel results. It is found that $\psi$ for infinite and finite aspect ratio channels coincide within the Stokes penetration depth which is $18 \%$ and $35 \%$ of the semi-diameter, but then the presence of the horizontal walls generates an steep increase up to approximately one quarter of the channel width where a plateau is reached. Even though the wall oscillation amplitude of the two cases analyzed differs by a factor of four, the maxima $\psi$ reached at $x=0$, are only $1.34 \pi$ and $1.22 \pi$ respectively. This observation contrasts with the corresponding values of $1.875 \pi$ and $0.875 \pi$ for the infinite channel. Figures (9), (10) and (12) indicate that in a relative small region near the channel center $(y=0)$ and close to the oscillatory walls $(x= \pm 0.5)$, the presence of the horizontal walls does not have a major influence on the flow, but elsewhere in the cavity, the limiting walls are determinant.

\subsection{Case $R e=500$}

Figures (13) to (15) show the velocity fields and vortices cores for $R e=500 Y=0.2,0.4$ and 0.8 , for two phases of the oscillation. The vortex distribution along the cycle is more complex than that for $R e=50$. The vortices generated by M1 are thinner that those observed in the previous subsection. The vortices generated by M2 are separated from those generated by M1. They are formed initially in the regions next to the moving walls as these meet the upper fixed walls (bottom fixed walls) at the phase of maximum wall velocity $\phi=\pi / 2$ $(\phi=3 \pi / 2)$. The maximum vorticity is seen to occur at $\phi=0(\phi=\pi)$ when the velocity of the sliding walls is zero. These vortices are labeled "A" in figure (15) and were described and interpreted by Tabaczynski et al. [4]. For $\mathrm{Y}=0.2$, the vortices dissipate at the core of the cavity, but for $\mathrm{Y}=0.4$ and 0.8 , they persist up to the phase $\phi=\pi / 2(\phi=3 \pi / 2)$ as indicated by label "B" in figure (15). Eventually, they dissipate in the core of the cavity. The presence of these structures at phases where the sliding walls move away from the fixed walls was not noticed in the visualizations made by Tabaczynski et al. [4].

The separation of the vortices formed by mechanism $M 2$ from the fixed wall that is observed on the flows described in this and the previous section, can be interpreted in terms of general concepts of interactions 

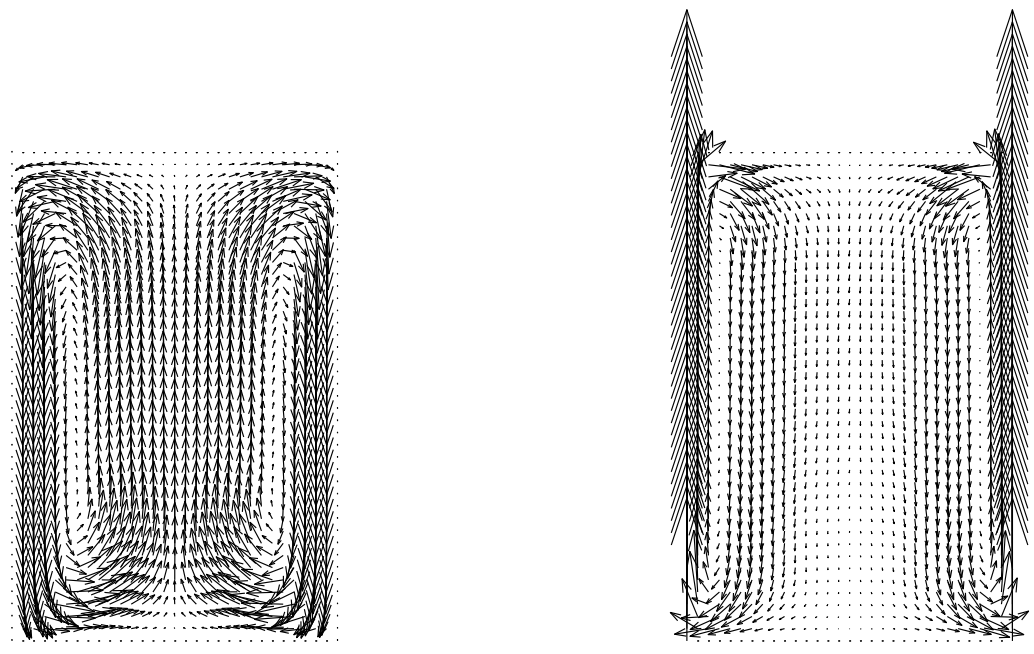

$\phi=0$

$\phi=\pi / 2$
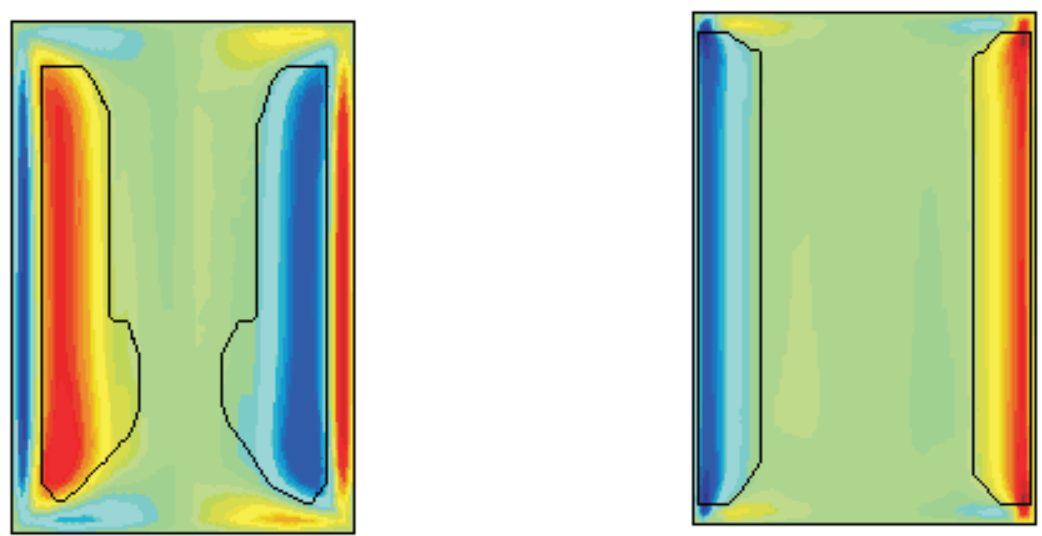

Figure 6: Velocity fields and vortices cores for $\phi=0$ and $\phi=\pi / 2$ with $R e=50$ and $Y=0.2$. 

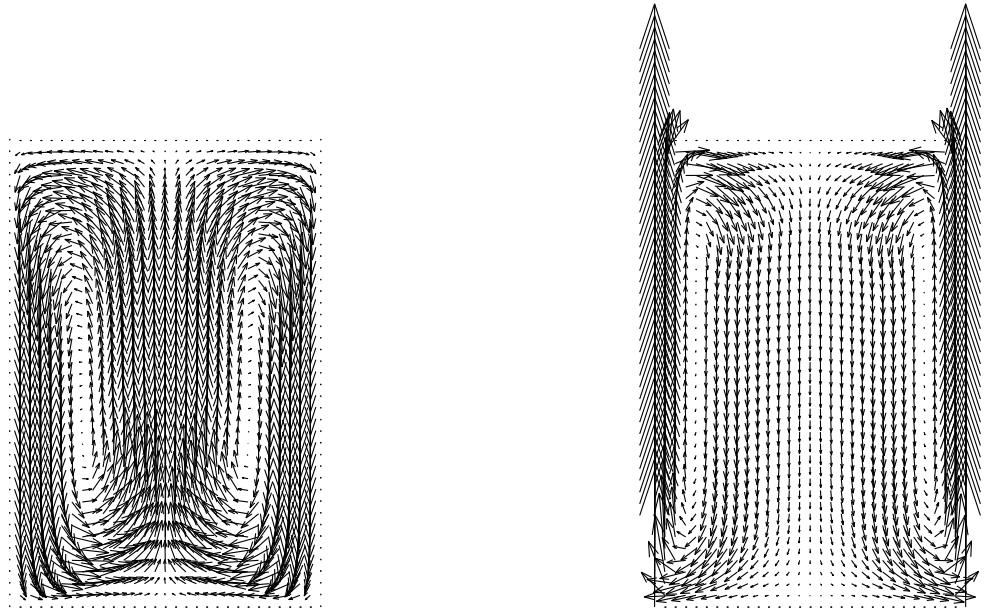

$\phi=0$

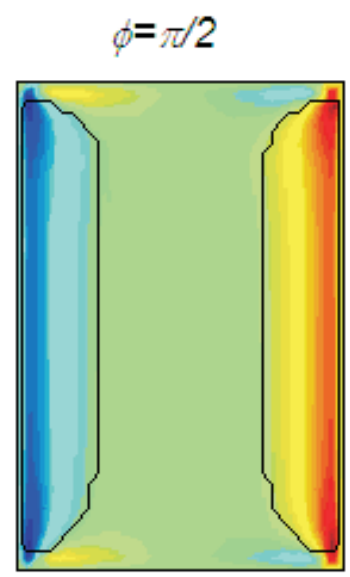

Figure 7: Velocity fields and vortices cores for $\phi=0$ and $\phi=\pi / 2$ with $R e=50$ and $Y=0.4$. 

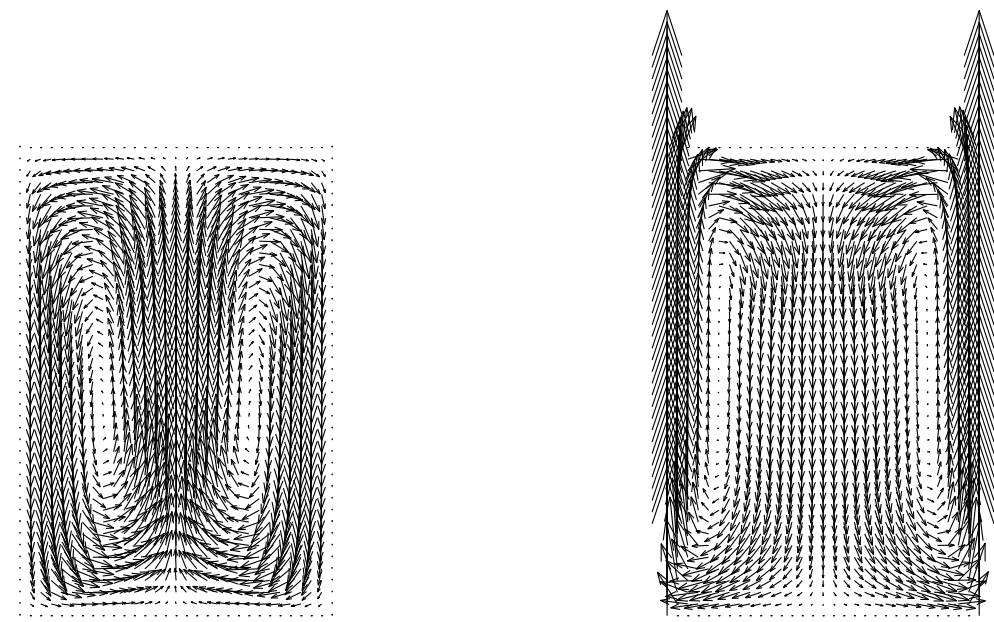

$\phi=0$

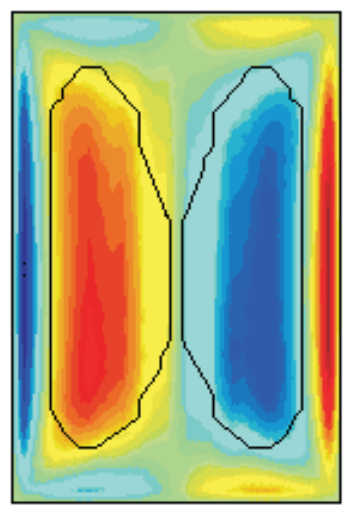

$\phi=\pi / 2$

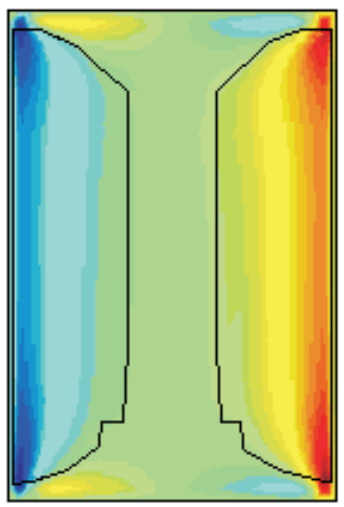

Figure 8: Velocity fields and vortices cores for $\phi=0$ and $\phi=\pi / 2$ with $R e=50$ and $Y=0.8$. 


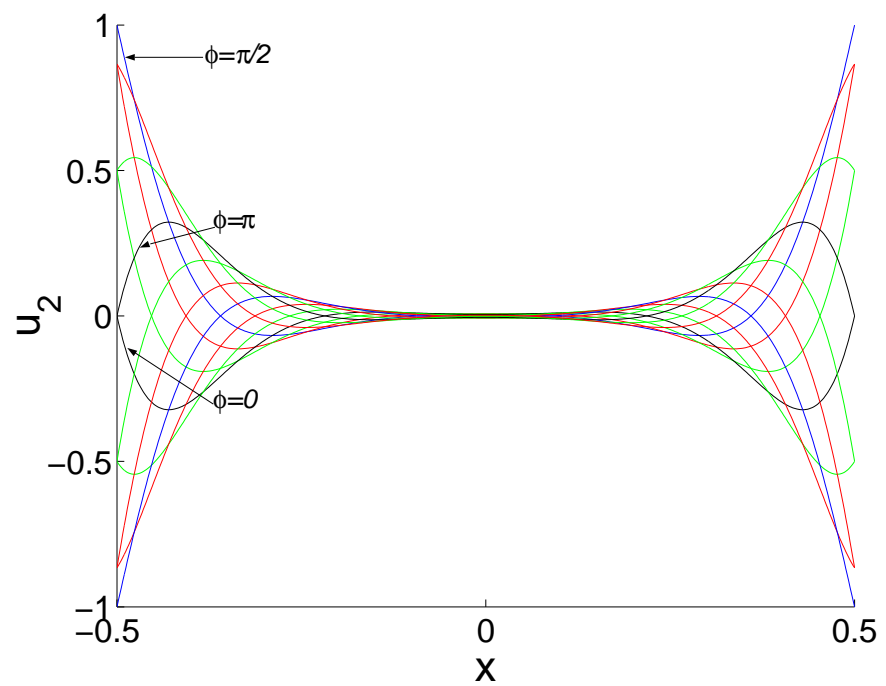

Figure 9: Axial velocity $u_{2}$ as a function of the transversal coordinate $x$ for an infinite channel (equation (15)) with $R e=50$ and $Y=0.2$. The phase interval between profiles is $\pi / 6$.

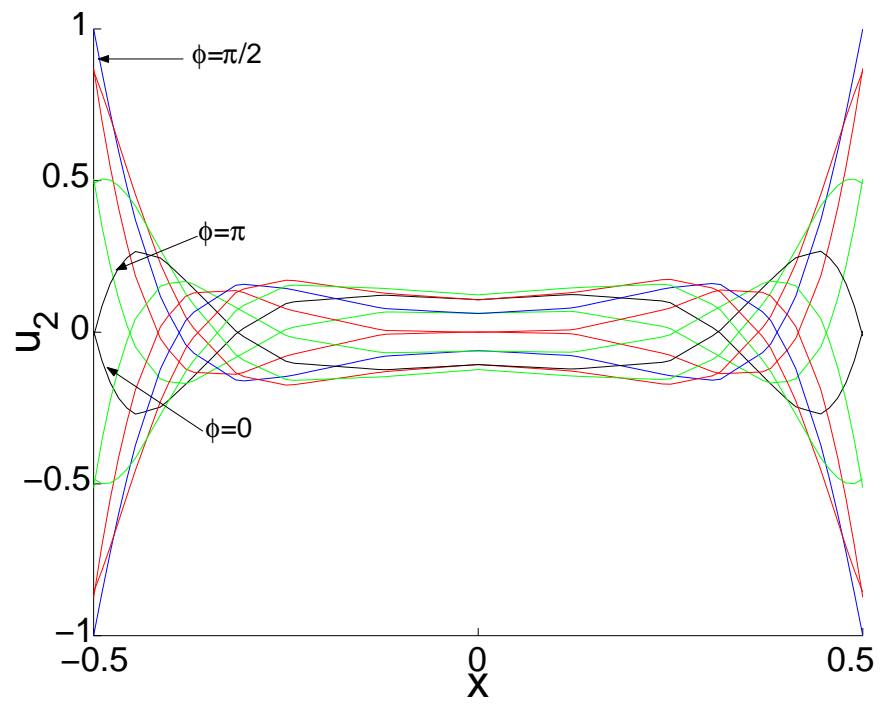

Figure 10: Axial velocity $u_{2}$ as a function of the transversal coordinate $x$ for a channel with aspect ratio $(H / D=1.5), R e=50$ and $Y=0.2$. For all profiles, $y=0$. The phase interval between profiles is $\pi / 6$. 


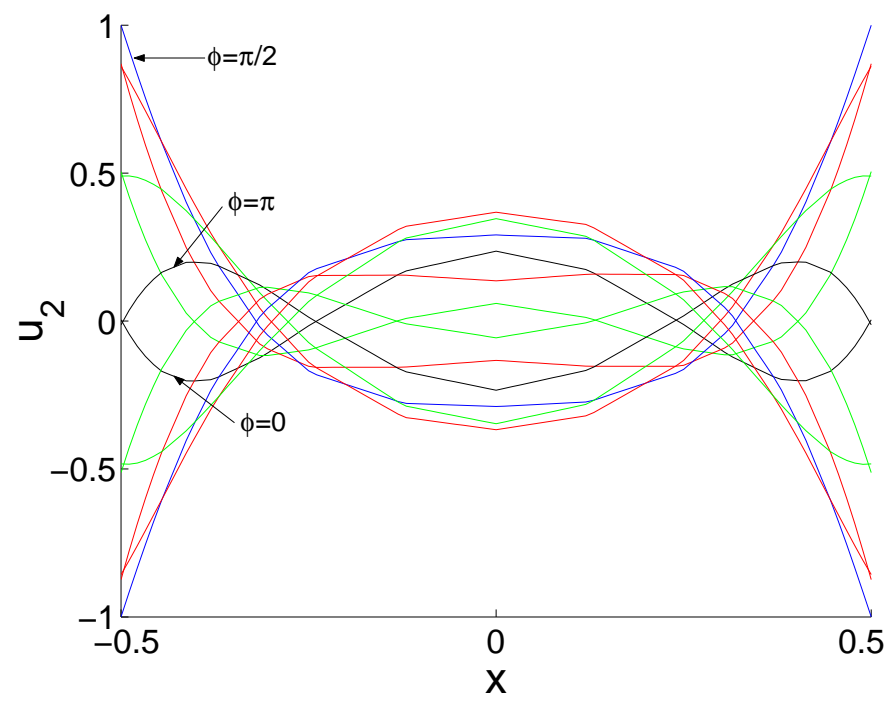

Figure 11: Axial velocity $u_{2}$ as a function of the transversal coordinate $x$ for a channel with aspect ratio $(H / D=1.5), R e=50$ and $Y=0.8$. For all profiles, $y=0$. The phase interval between profiles is $\pi / 6$.



Figure 12: Relative phase between oscillating walls and the fluid axial velocity $\psi$ as a function of the transversal coordinate $x . R e=50$ and $y=0$. 
of individual vortices and walls. It has been found that as a vortex approaches a wall perpendicular to its direction of motion, a region with opposite vorticity is formed between its core and the wall. See for instance Walker et al. [28] and Allen and Chong [7] for experimental observations and Peace and Riley [30] for numerical calculations. In our simulations, this effect is present at the lower fixed wall for $\phi=0$ as can be seen from figure (16) where the vorticity field is plotted as a function of position for $R e=500, Y=0.8$. As the vortices moving downwards approach the horizontal wall, a secondary vorticity region with opposite sign is formed between the vortex core and the fixed wall, inducing the vortex to move away from the fixed wall. Given that the driving force of the flow is oscillatory, the formation of the vortices and the induced secondary vorticity regions are periodic too.

The pattern observed by the visualization of vortices generated by the oscillatory flow for $R e=500$, $Y=0.8$, is shown in figure (17). The streak lines in this figure simulate a tracer injected at approximately one tenth of the height $(y=-0.610)$ and $-0.450<x<-0.423$. Injection starts when the velocity is maximum positive and displacement is zero $(\phi=\pi / 2)$, and it lasts throughout the one full cycle simulation. It is seen that the pattern formed with this visualization technique has three zones. The first comprises the tracer that is been injected near the end of the cycle and the tracers are distributed near the sliding wall. The second is an spiral similar to that observed in experiments where the velocity of the wall is unidirectional as shown by Tabaczynski et al. [4] and Allen and Chong [7]. In the present case however, the traces do not wind up tightly at the center of the spiral but rather form another structure where all tracers are distributed close together that contains the spiral. This complex distribution of tracers are a consequence of the oscillatory motion of the sliding wall.

\subsection{Case $R e=1000$}

Figures (18) and (19) show the velocity fields and vortices cores for $Y=0.2$ and 0.4 , for two phases of the oscillation. The vortex distribution and evolution are similar to those of $R e=500$, only the vortical structures are thinner and confined to the regions near the walls. Most of the channel is vortex free all along the cycle. As the amplitude of the displacement is increased, the area occupied by the vortices is enlarged, but the qualitative properties of the flow are the same. See figure (19). In all cases presented up to this point, the flow distributions display the axial and cyclic symmetries defined at the beginning of section 4 . When the amplitude of the wall oscillation is increased to $Y=0.8$, the symmetry around $x=0$ is lost. Figure (20) shows the velocity fields and vortices cores for $Y=0.8$, for two phases of the oscillation. With this set of parameters, the flow is not axial symmetric anymore but it conserves cycle symmetry. At $\phi=0$, the right and left vortices generated as the sliding walls approach the bottom wall and those near the upper wall are not of the same size. This imbalance generates a cross flow that moves from the bottom right corner to the upper left corner of the cavity forming a large vortex rotating clockwise. See upper left panel in figure (20). The vortex distribution shown in the lower left panel of figure (20) confirm the previous observations by 

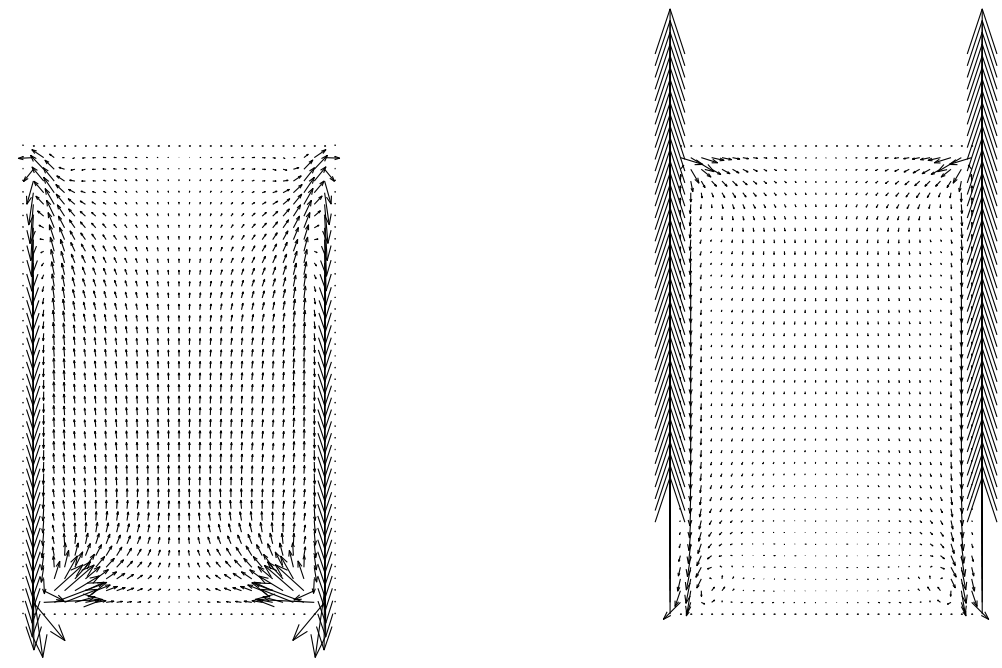

$\phi=0$

$\phi=\pi / 2$
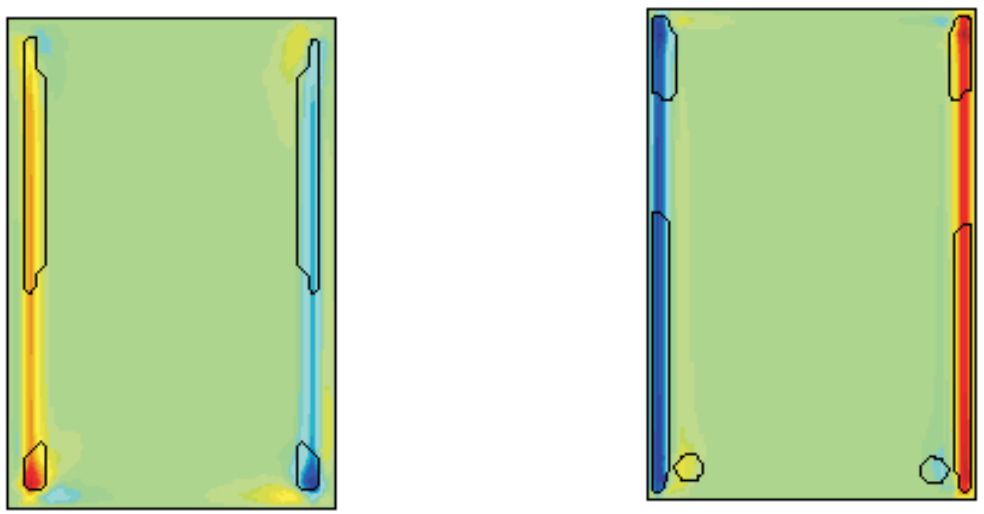

Figure 13: Velocity fields and vortices cores for $\phi=0$ and $\phi=\pi / 2$ with $R e=500$ and $Y=0.2$. 


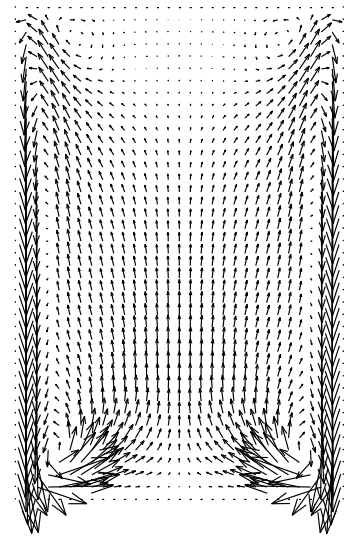

$\phi=0$

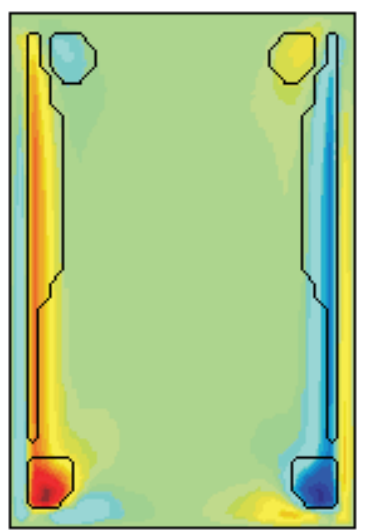

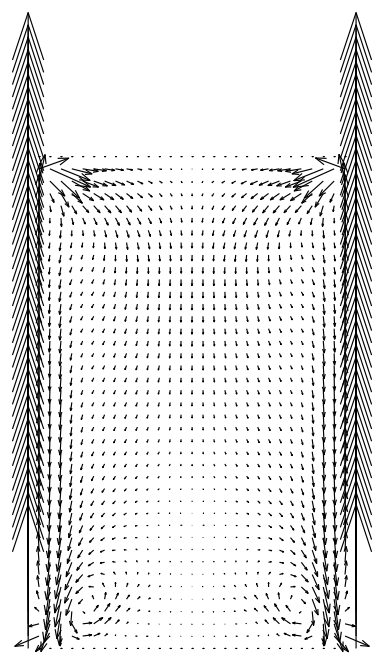

$\phi=\pi / 2$

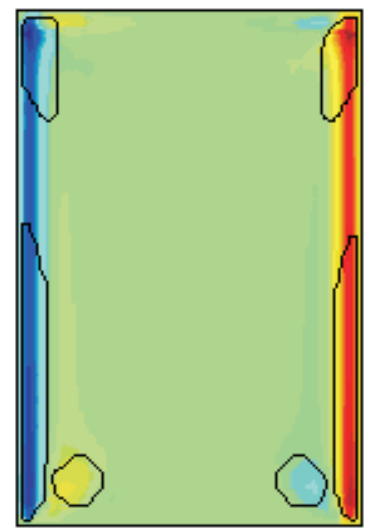

Figure 14: Velocity fields and vortices cores for $\phi=0$ and $\phi=\pi / 2$ with $R e=500$ and $Y=0.4$. 


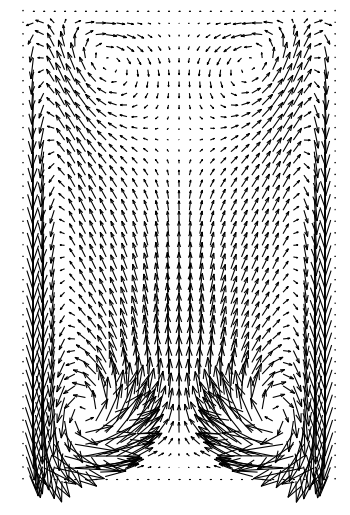

$\phi=0$



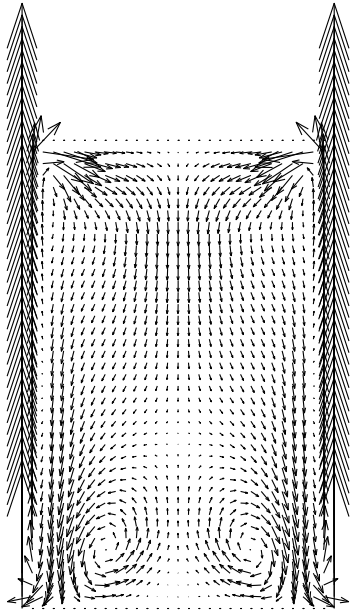

$\phi=\pi / 2$

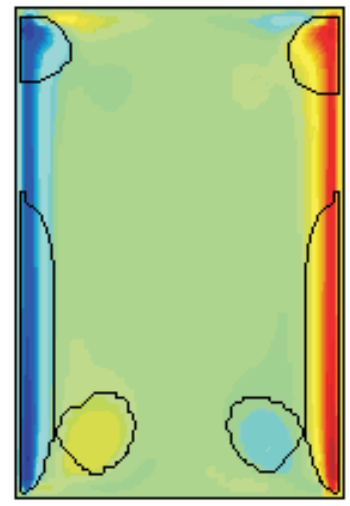

Figure 15: Velocity fields and vortices cores for $\phi=0$ and $\phi=\pi / 2$ with $R e=500$ and $Y=0.8$. 
$\phi=0$
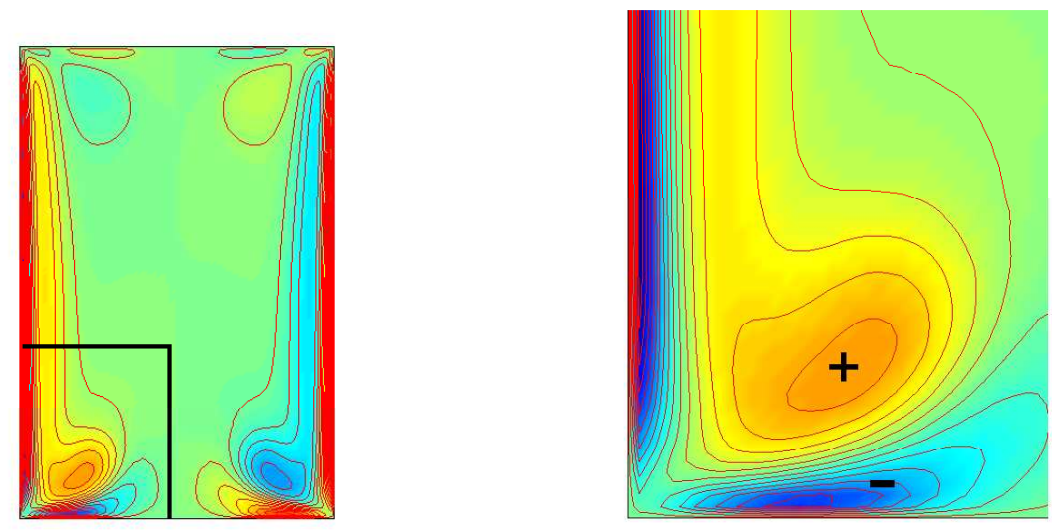

Figure 16: Vorticity field as a function of position for $R e=500, Y=0.8, \phi=0$. The + and - signes indicate positive and negative vorticities, respectively.

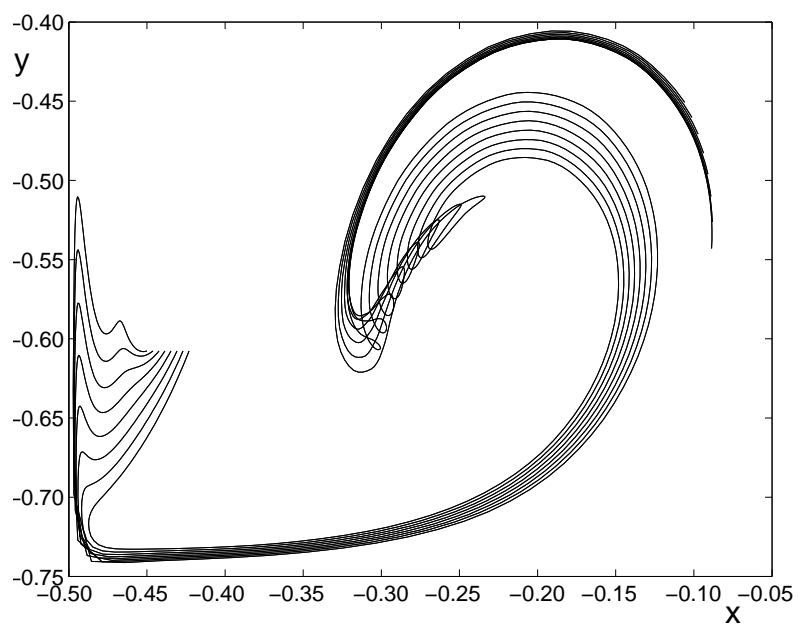

Figure 17: Streak lines for $\mathrm{Re}=500, \mathrm{Y}=0.8$ to simulate flow visualization. Tracer injection starts at $\phi=\pi / 2$ and continues for one cycle. Coordinates at for tracer injection are $x=-0.450,-0.446,-0.442,-0.439$, $-0.435,-0.431,-0.427$ and $-0.423, y=-0.610$. 
displaying a large structure near the center of the cavity. It is instructive to compare these figures with the corresponding for $R e=500$ given in figure (15) which can be interpreted as an approximate symmetric version of (20) where the cross flow and the central vortex are absent. The flow structure at $\phi=\pi / 2$ (right hand side panels of figure (20)) also includes a large central vortex and is similar to that of $\phi=0$. Left upward and right downward flows are more intense. The governing equations and boundary conditions are symmetric with respect to the $x=0$ axis, therefore, a velocity and pressure fields of the form:

$$
\begin{gathered}
u_{1}(x, y, \phi)=-u_{1}^{*}(-x, y, \phi), \quad u_{2}(x, y, \phi)=u_{2}^{*}(-x, y, \phi), \\
p(x, y, \phi)=p^{*}(-x, y, \phi),
\end{gathered}
$$

where $u_{1}^{*}, u_{2}^{*}$ and $p^{*}$ are the velocity and pressure distributions of the flow described in the previous paragraph and illustrated in figure (20), is also a solution. We conclude then that there is a qualitative change in the dynamical behavior featured by the breaking of the axial symmetry, which for $R e=1000$ occurs between $0.4<Y<0.8$, and for $Y=0.8$, it occurs within $500<R e<1000$. We analyze this breakdown in section 4.5 .

Figure (21) shows the profile of axial velocity $u_{2}$ for a channel with infinite length as a function of the transversal coordinate $x$. In this figure, $R e=1000$ and $Y=0.2$. The Stokes penetration depth is smaller and a thinner boundary layer forms near the wall. The core of the channel is motionless. The corresponding plots of axial velocity for a channel with aspect ratio 1.5 and $R e=1000$ are displayed in figures (22) and (23) for $Y=0.2$ and 0.8 respectively. The plots in these figures are obtained for $y=0$. The properties of the velocity profiles for $Y=0.2$ (figure(22)) are similar to those described for the cases of section 4.2 namely, the profiles for an infinite channel and a finite aspect ratio channel are indistinguishable near the walls $\left((D / 2) / \delta_{\nu}=25\right)$.

The boundary layer is thinner than that obtained for smaller Reynolds numbers and the flow in the core is not stagnant but ascends or descends at different phases of the oscillation. Also, it was found (but not shown in figures) that, as discussed in section 4.2, the distortion due to the presence of the horizontal walls in the central part of the cavity increase as $Y$ increases.

For $Y=0.8$ we observe notable differences in the velocity distributions with respect to the infinite channel behavior due to the breaking of the axial symmetry, as is shown in figure (23). It is found that a clockwise vortex is permanently present in the cavity, deflecting slightly upwards the velocity traces on the left side and downwards near the right side. Velocities close to the moving walls $\left((D / 2) / \delta_{\nu}=12.5\right)$ are symmetric, but in the core they are distorted due to the vortical global motion which has always the same direction of rotation. As commented previously, a second solution also exists in which the core vortex rotates anticlockwise.

\subsection{Axial symmetry breakdown analysis}

To study the axial symmetry breakdown of the steady state, we consider $Y=0.8$ and take $R e$ as the bifurcation parameter. The nonlinear criticality of the axial symmetry bifurcation is determined by examining 


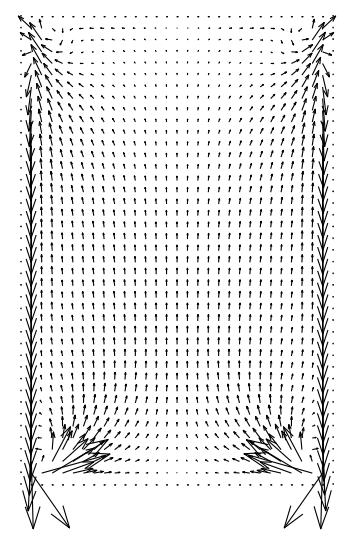

$\phi=0$

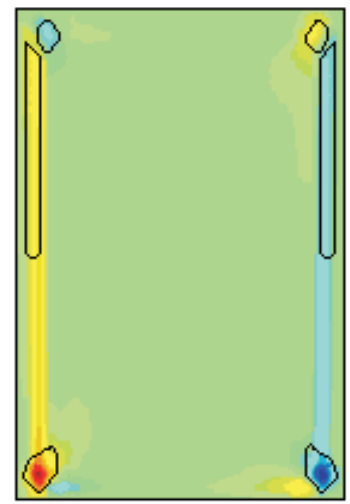

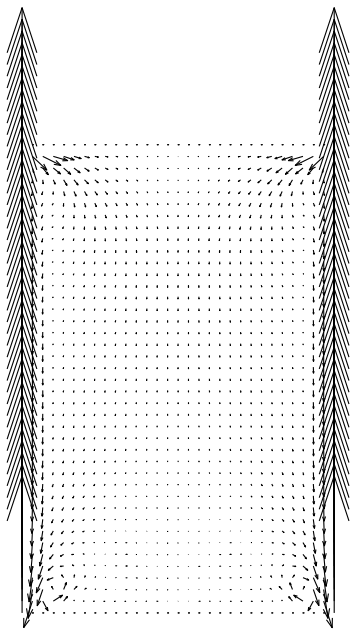

$\phi=\pi / 2$

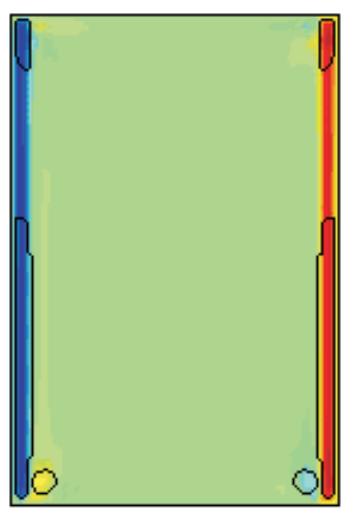

Figure 18: Velocity fields and vortices cores for $\phi=0$ and $\phi=\pi / 2$ with $R e=1000$ and $Y=0.2$. 



$\phi=0$

$\phi=\pi / 2$
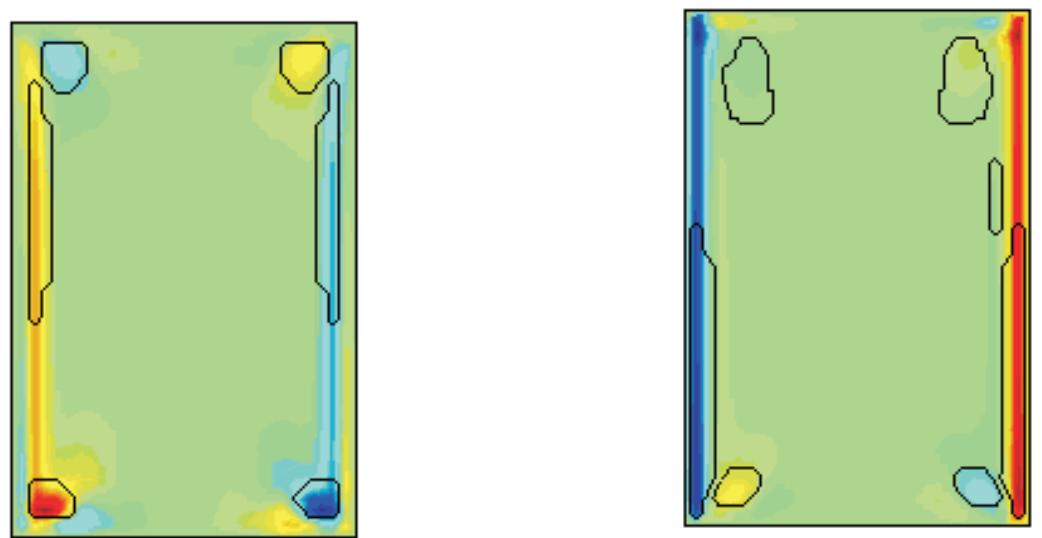

Figure 19: Velocity fields and vortices cores for $\phi=0$ and $\phi=\pi / 2$ with $R e=1000$ and $Y=0.4$. 

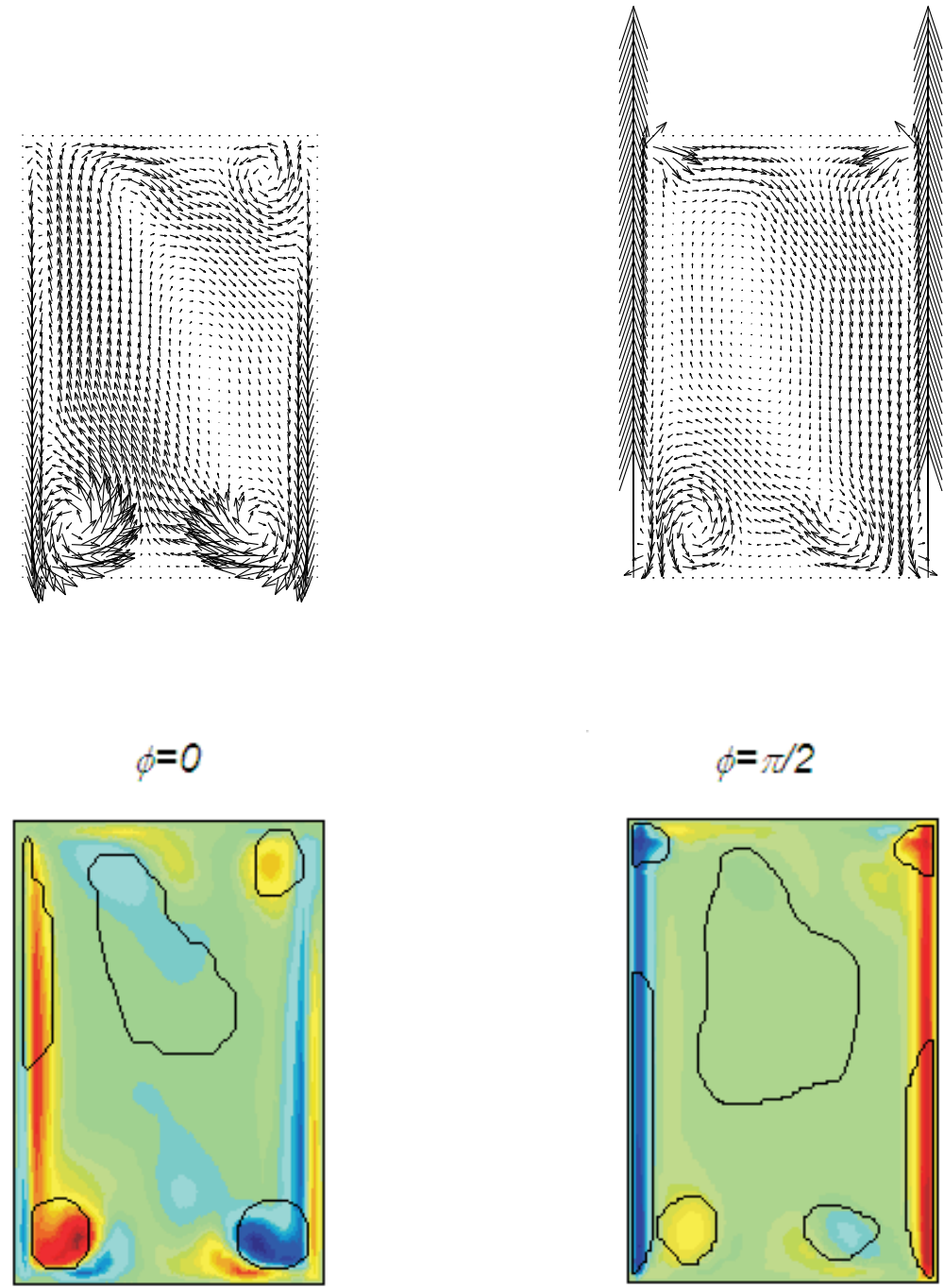

$$
\phi=\pi / 2
$$

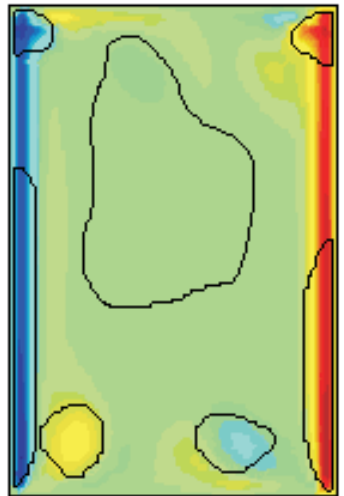

Figure 20: Velocity fields and vortices cores for $\phi=0$ and $\phi=\pi / 2$ with $R e=1000$ and $Y=0.8$. 


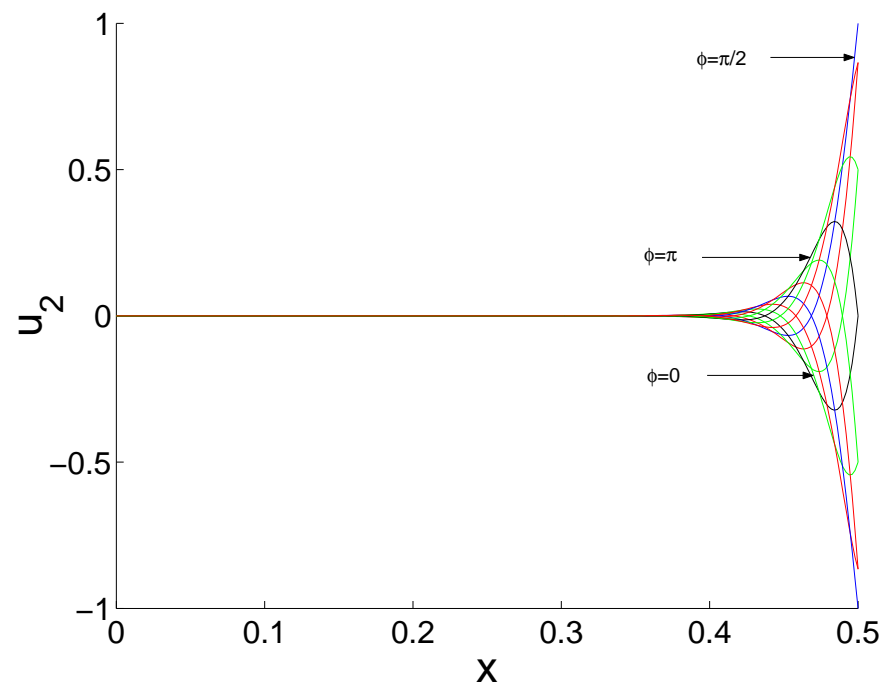

Figure 21: Axial velocity $u_{2}$ as a function of the transversal coordinate $x$ for an infinite channel (equation (15)) with $R e=1000$ and $Y=0.2$. Phase intervals between profiles are $\Delta \phi=\pi / 6$.

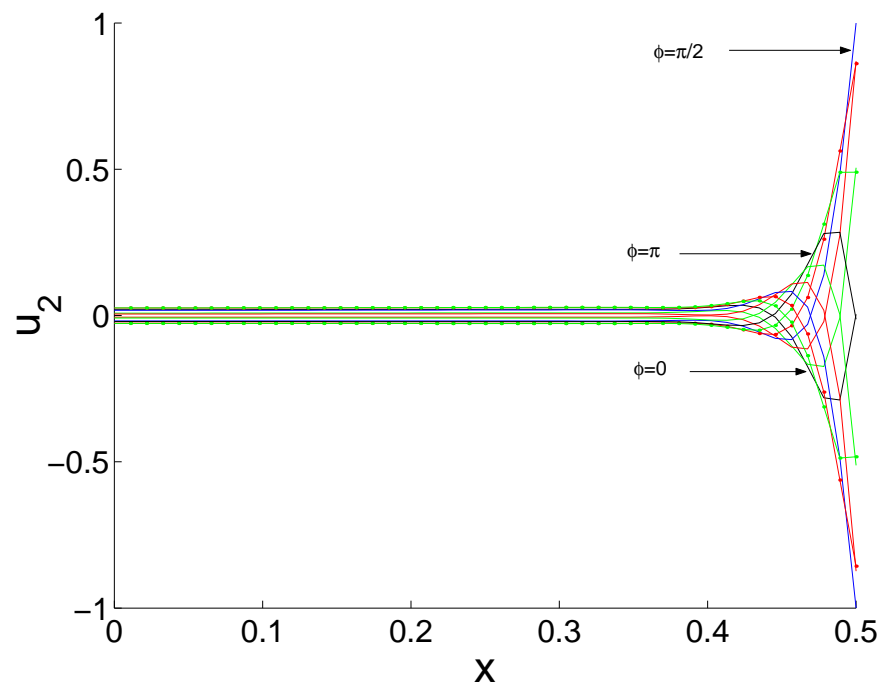

Figure 22: Axial velocity $u_{2}$ as a function of the transversal coordinate $x$ for a channel with aspect ratio $(H / D=1.5), R e=1000$ and $Y=0.2$. For all profiles, $y=0$. Phase intervals between profiles are $\Delta \phi=\pi / 6$. 




Figure 23: Axial velocity $u_{2}$ as a function of the transversal coordinate $x$ for a channel with aspect ratio $(H / D=1.5), R e=1000$ and $Y=0.8$. For all profiles, $y=0$. Phase intervals between profiles are $\Delta \phi=\pi / 6$.

the variation with $R e$ of a measure related to the energy (squared amplitude) of the asymmetry of the flow [9]. Here, this measure is defined as

$$
Q=\int_{-H / 2}^{H / 2} \int_{-\pi \omega}^{\pi \omega} u_{1}^{2}(x=0, y, t) d t d y .
$$

Clearly, $Q$ is zero for symmetric flows. Steady state is defined when the percentage difference between $Q$ for a cycle and the previous cycle is less than 0.01. Figure 24 shows $Q$ as a function of $R e$, symbols are simulation results and the line is the linear fit to the first four points above $R e_{c}=669.1 \pm 0.5$. This mathematical relationship between $Q$ and $R e$ near $R e_{c}$ indicates that the symmetry is broken through a supercritical pitchfork bifurcation.

\section{Discussion and Conclusions}

A numerical solution of the Navier-Stokes equations, using finite element and an operator splitting scheme, have been obtained for simulating the flow in a cavity with vertical walls simultaneously oscillating. In contrast with others studies [31] and [32], using our numerical method, we were able to predict the formation of vortices without introducing any random perturbation. For the range of oscillation amplitude $Y$ and Reynolds number $R e$ explored, we have found that the main feature of the flow is the formation of vortices with cyclic symmetry. For low values of $Y$ and $R e$, the vortices also present symmetry with respect to the vertical axis dividing the cavity in two sides. In asymmetric flows, the unbalance between the vortices on each side of the mid-vertical line generates a vortex that occupies the central part of the cavity and preserves vorticity sign along the cycle. The vorticity sign of the cental vortex depends on initial conditions. 


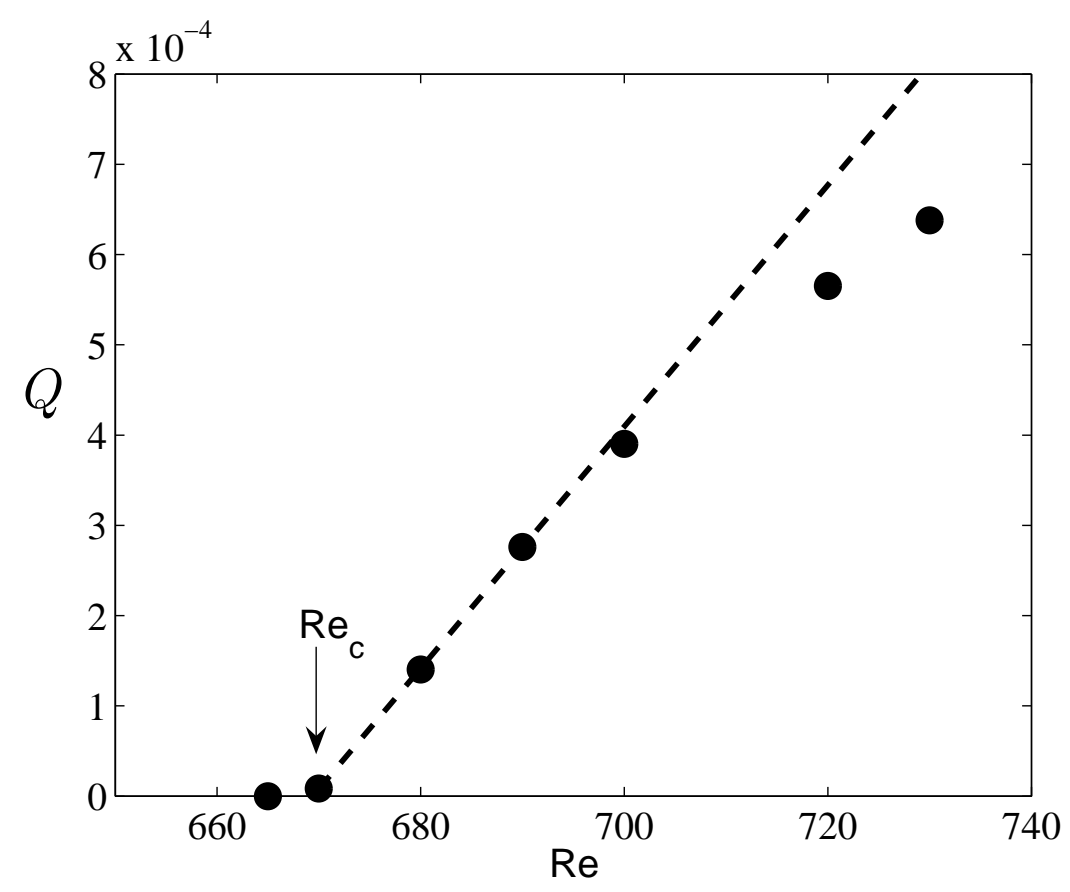

Figure 24: $Q$ as a function of $R e$, symbols are simulation results and the line is the linear fit to the first three points above $R e_{c}=669.1 \pm 0.5$

We have identified two basic mechanisms of vortex formation: The first is vorticity injection into the fluid due to the shear motion of the moving walls in the presence of the fixed walls that provide a translational symmetry breaking (M1). Note that both fixed walls break the translational symmetry, regardless of whether they are up- or down-stream the flow and thus contribute to the formation of vortices. These vortices are elongated, aligned with the oscillatory walls with return regions near the fixed walls and change their vorticity sign along the cycle. Their characteristic dimension in the transversal direction is inversely proportional to the frequency of oscillation which for fixed fluid and geometry can be expressed in terms of the oscillatory Reynolds number defined by $R_{\omega}=R e / Y=\omega D^{2} / \nu$. Note for instance that the width of the elongated vortices cores of figures $(Y=0.2, R e=500)$ and $(Y=0.4, R e=1000)$ at $\phi=0$ which have the same oscillatory Reynolds number are equal. The second vortex formation mechanism is the abrupt change in the flow direction as the fluid meets the downstream fixed wall (M2). This effect occurs for oscillatory walls as well as for a walls moving with constant velocity and is a consequence of boundary layer being scrapped off by the fixed wall. Since the flow under analysis is oscillatory, the vortex formation by this mechanism is strongly dependent on the phase of the cycle. Vortices are formed at the bottom (top) fixed wall at $\phi=0(\pi)$ when the flow moves against the wall. The vortices formed by mechanism M2 conserve their vorticity sign and remain at phases where the sliding walls move away from the fixed wall, but detach from the corresponding moving wall. We found that for Reynolds number greater than 500 the detach of vortices by mechanism 
M2 occurs, this agrees with the critical Reynolds number $(>450)$ for the vortex ring formation obtained by Hughes and Gerrard [32] and with the Reynolds number ( $>400$ ) for the roll up of vortices obtained by Allen and Chong [7].

The streak lines topology are difficult to analyze since for this time-dependent flow, the trace distributions depend on the position where the traces are released, the phase in the cycle when this occurs and the duration. We have just illustrated one case to highlight the fact that these features might be interesting in the context of mixing with oscillatory flows.

The breakdown of the axial symmetry was studied for a fixed value of the oscillation amplitude, $Y=0.8$, taking $R e$ as the bifurcation parameter. The results indicates that the symmetry is broken through a supercritical pitchfork bifurcation close to a degenerate case.

show that All the analyzed cases display cyclic symmetry and in contrast with all other cases analyzed, for $Y=0.8$ and $R e=1000$, the distribution of vortices is not symmetric with respect to the vertical axis. The is due to the formation of a large vortex in the center of the cavity rotating in the same direction in the whole cycle. This change in the dynamical behavior is the subject of ongoing research.

Our results can also be described in terms of the Stokes parameter $S t=\omega H^{2} / \nu$. The cases explored in the present investigation are $\mathrm{Re}=50: \mathrm{St}=10,20,40 ; \mathrm{Re}=500: \mathrm{St}=100,200,400$ and $\mathrm{Re}=1000: \mathrm{St}=200,400,800$. The smallest values of the Stokes parameter correspond to the largest values of Y. The non-symmetric flow described in detail corresponds to $\mathrm{Re}=1000, \mathrm{St}=200$. It is interesting to observe that all cases studied fall in the region where stable, two-dimensional flow is predicted by the theory for three-dimensional instabilities of the two-dimensional flow in a rectangular cavity driven by the simple harmonic oscillation of one wall presented by Blackburn and Lopez (2003), but the case $\mathrm{Re}=1000, \mathrm{St}=200$ is closest to the neutral stability line. The model presented here considers only two dimensional flows and therefore is unable to determine whether the $2 \mathrm{D}$ instability would appear in a real system before a 3D instability develops. In order to address this point, a three dimensional model of the flow would be required, but unfortunately this is beyond the scope of the present investigation.

Although the application of the present study is limited due to the fact that is made for a two dimensional flow, it is considered that the time-dependent vortex distribution is potentially useful for understanding transport properties in oscillatory flows.

\section{Acknowledgements}

The authors thank Francisco Mandujano and Raúl Rechtman for fruitful discussions and suggestions. Partial economic support from DGAPA-UNAM and CONACyT under projects IN104702-2 and U41347-F, respectively, is acknowledged. GEOC thanks CONACyT for a postgraduate scholarship. 


\section{References}

[1] Lee D., Hochgreb S.: Rapid compression machines: heat transfer and suppression of corner vortex. Combustion and flame 114, 531-545 (1998)

[2] Lopez, J. M. and Hirsa, A.: Oscillatory driven cavity with an air/water interface and an insoluble monolayer: Surface viscosity effects. J. Colloid Interface Sci. 242, 1-5 (2001)

[3] O'Brien V.: Unsteady separation phenomena in a two-dimensional cavity AIAA J. bf 13, 415-416 (1975)

[4] Tabaczynski R. J., Hoult D. P., Keck J. C.: High Reynolds number flow in a moving corner. J. Fluid Mech. 42, 249-255 (1970)

[5] Taylor G.: Aeronautics and Aeromechanics. Pergamon (1960)

[6] Batchelor G. K.: An Introduction to Fluid Dynamics. Cambridge University Press (1967)

[7] Allen J. J., Chong M. S.: Vortex formation in front of a piston moving through a cylinder. J. Fluid Mech. 416, 1-28 (2000)

[8] Vogel M. J., Hirsa A. H., and Lpez J. M.: Spatio-temporal dynamics of a periodically driven cavity flow, J. Fluid Mech. 478, 197-226 (2003)

[9] Blackburn H. M., Lopez J. M.: The onset of three-dimensional standig and modulatedd travelling waves in a periodically driven cavity flow. J. Fluid Mech. 497, 289-317 (2003)

[10] Leung J. J. F., Hirsa A. H., Blackburn H. M., Marquez F., and Lopez, J. M.: Three-dimensional modes in a periodically driven elongated cavity, Physical Rev. E, 71, 026305(2005)

[11] Temam R.: Theory and numerical analysis of the Navier-Stokes equations. North-Holland, Amsterdam (1977)

[12] Girault V., Raviart P.A.: Finite element methods for the Navier-Stokes equations: theory and algorithms. Springer-Verlag, Berlin (1986)

[13] Gunzburger M. D.: Finite element methods for viscous incompressible flows. Academic Press, Boston MA (1989)

[14] Glowinski R.: Numerical methods for fluids. part 3, Handbook of Numerical Analysis, vol IX, P. G: Garlet and J. L. Lions eds., North-Holland, Amsterdam (2003)

[15] Bercovier M., Pironneau O.: Error estimates for the finite element solution of the Stokes problem in the primitive variables. Numer. Math. 33, 211-224 (1979) 
[16] Chorin A.: Numerical study of slightly viscous flow. J. Fluid Mech. 57, 785-796 (1973)

[17] Glowinski R., Pironneau O.: Finite element methods for the Navier-Stokes equations. Annual rev. Fluid Mech. 24, 167-204 (1992)

[18] Turek S.: Efficient solvers for incompressible flow problems: An algorithmic and computational approach. Springe-Verlag, Berlin (1999)

[19] Marechuk G. I.: Splitting and alternate direction methods. in Handbook of Numerical Analysis, vol 1, P.G. Garlet and J.L. Lions eds., North-Holland, Amsterdam 197-462 (1990)

[20] Beale J. T., Majda A.: Rates of convergence for viscous splitting of the Navier-Stokes equations. Math. Comput. 37, 243-260 (1981)

[21] Dean E. J., Glowinski R.: A wave equation approach to the numerical solution of the Navier-Stokes equations for incompressible viscous flow. C. R. Acad. Sci. Paris, 325, Serie I, 789-797 (1997)

[22] Glowinski R., Le Tallec P.: Augmented Lagrangian and operator-splitting methods in nonlinear mechanics. SIAM, Philadelphia (1989)

[23] Press W. H., Teukolsky S. A., Vetterling W. T., Flannery B. P.: Numerical recipes in C++. Cambridge University Press, Cambridge (2002)

[24] Pironneau O.: Finite element methods for fluids. Wiley, Chichester (1989)

[25] Dean E. J., Glowinski R., Pan T. W.: A wave equation approach to the numerical simulation of incompressible viscous fluid flow modeled by the Navier-Stokes equations. Mathematical and numerical aspects of wave propagation. Edited by J. A. de Santo, SIAM, Philadelphia, 65-74 (1998)

[26] Jeong J., Hussain F.: On the identification of a vortex. J. Fluid Mech. 285, 69-94 (1995)

[27] Currie I. G.: Fundamental mechanics of fluids. McGrawHill, New York (1974)

[28] Walker J., Smith C., Cerra A., Doligalski T. The impact of a vortex ring on a wall. J. Fluid Mech. 181, 99-140 (1987)

[29] Allen J. J., Auvity B.: Interaction of a vortex ring with a piston vortex. J. Fluid Mech. 465, 353-378 $(2002)$

[30] Peace A. J., Riley N.: A viscous vortex pair in ground effect. J. Fluid Mech. 129, 409-426 (1983)

[31] Gerrard J. H.: The stability of unsteady axisymmetric incompressible pipe flow close to a piston. Part 1. Numerical analysis. J. Fluid Mech. 50, 625-644 (1971) 
[32] Hughes M. D., Gerrard J. H.: The stability of unsteady zxisymmetric incompressible pipe flow close to a piston. Part 2. Experimental investigation and comparison with computation. J. Fluid Mech. 50, 645-655 (1971) 